\title{
Predictors of Subsequent Heart Failure After Left Atrial Appendage Closure
}

\author{
Dae-Young Kim, MD; Min Ji Kim, MD; Jiwon Seo, MD; \\ Iksung Cho, MD; Chi Young Shim, MD, PhD; Geu-Ru Hong, MD, PhD; \\ Jung-Sun Kim, MD, PhD; Jong-Won Ha, MD, PhD
}

\begin{abstract}
Background: Left atrial appendage occlusion (LAAO) plays an important role in preventing stroke in patients with atrial fibrillation. However, LAAO may interact unfavorably with left atrial (LA) compliance and reservoir function and thus increase the risk of heart failure (HF). The purpose of this study was to identify predictors of subsequent HF after successful LAAO.
\end{abstract}

\begin{abstract}
Methods and Results: A total of 98 patients (mean age $70 \pm 9$ years, $68 \%$ male) who had undergone LAAO were included. The primary endpoint was unexpected HF admission after LAAO. During a mean period of $36 \pm 26$ months, 16 of the 98 patients (16\%) experienced hospital HF admission. In multivariate analysis, higher E/e' (hazard ratio [HR] 1.11, 95\% confidence interval [Cl] 1.02-1.20, $\mathrm{P}=0.014$ ), higher left ventricular mass index ( $\mathrm{HR} 1.02,95 \% \mathrm{Cl} 1.00-1.03, \mathrm{P}=0.023$ ), history of $\mathrm{HF}$ ( $\mathrm{HR} 4.78,95 \% \mathrm{Cl} 1.55-14.7, \mathrm{P}=0.006$ ), and lower LA strain ( $\mathrm{HR} \mathrm{0.80,95 \%} \mathrm{Cl} 0.70-0.93, \mathrm{P}=0.003$ ) were independently associated with hospital HF admission. Patients with LAAO had a significantly higher incidence of subsequent HF than the control group after propensity score matching $(P=0.046)$.
\end{abstract}

Conclusions: LAAO increases the occurrence of HF, and it is not uncommon after successful LAAO. A previous history of HF, left ventricular mass index, E/e', and abnormal LA strain are independently associated with the development of HF. These parameters should be considered before attempting LAAO.

Key Words: Atrial fibrillation; Diastolic; Echocardiography; Heart failure; Stroke

A trial fibrillation (AF) is the most common arrhythmia worldwide, ${ }^{1,2}$ and anticoagulation is a major treatment strategy for preventing stroke in patients with AF. ${ }^{3,4}$ Because the majority of AF-related left atrial (LA) thrombi are located in the LA appendage (LAA), 5 LA appendage occlusion (LAAO) emerged as an important therapeutic strategy for preventing stroke and is beneficial for patients who are not able to maintain oral anticoagulation due to a higher risk of bleeding. ${ }^{7,8}$

In contrast, the LAA has the reservoir function of absorbing pressure and distending its chamber when LA volume and pressure rises; ${ }^{9}$ therefore, exclusion of the LAA may result in unfavorable LA pressure-volume relations and worsening LA compliance. ${ }^{10}$ However, the development and aggravation of heart failure (HF) was not consistently assessed in previous trials on LAAO. The purpose of this study was to identify the predictors of subsequent HF after successful LAAO.

\section{Methods}

\section{Study Population}

Patients with AF who underwent LAAO between October
2010 and March 2020 and who had baseline transthoracic echocardiography (TTE) were included retrospectively in a single tertiary center study. The following conditions were excluded from the study: no baseline TTE prior to LAAO and history of surgical closure of LAA. Patient clinical data, echocardiographic characteristics, and outcomes were retrospectively reviewed. To reduce the confounding factors in patients with LAAO, subgroup analysis was performed comparing patients with LAAO against controls without LAAO, matched by propensity score. From October 2010 to March 2020, patients who had a history of non-valvular AF with a $\mathrm{CHA}_{2} \mathrm{DS}_{2}-\mathrm{VASc}$ score of at least 2 and met any of the following criteria: (1) any history of significant bleeding; (2) increased risk of bleeding as indicated by a HAS-BLED score of at least 3; and (3) history of thromboembolic events despite adequate oral anticoagulants, were finally selected for propensity score matching. Among those patients, the propensity scores were estimated based on 1-to-1 nearest-neighbor matching. Age, sex, hypertension, diabetes mellitus, HF, chronic kidney disease, ischemic stroke, coronary or peripheral vascular disease, $\mathrm{CHA}_{2} \mathrm{DS}_{2}$-VASc score, left

Received July 20, 2021; revised manuscript received September 24, 2021; accepted November 4, 2021; J-STAGE Advance Publication released online December 8, 2021 Time for primary review: 20 days

Division of Cardiology, Severance Cardiovascular Hospital, Yonsei University College of Medicine, Seoul, Korea

Mailing address: Jong-Won Ha, MD, PhD, FESC, Division of Cardiology, Severance Cardiovascular Hospital, Yonsei University College of Medicine, Yonsei-ro 50-1, Seodaemun-gu, Seoul 03722, Korea. E-mail: jwha@yuhs.ac

All rights are reserved to the Japanese Circulation Society. For permissions, please e-mail: cj@j-circ.or.jp

ISSN-1346-9843 


\begin{tabular}{|c|c|c|c|c|}
\hline & $\begin{array}{c}\text { Total } \\
(\mathrm{N}=98)\end{array}$ & $\begin{array}{l}\text { Without hospital } \\
\text { HF admission } \\
(\mathrm{N}=82)\end{array}$ & $\begin{array}{l}\text { With hospital } \\
\text { HF admission } \\
(\mathrm{N}=16)\end{array}$ & $P$ value \\
\hline Age, years & $70.2 \pm 9.0$ & $70.9 \pm 7.9$ & $66.8 \pm 13.1$ & 0.249 \\
\hline Male sex, $\mathrm{n}(\%)$ & $67(68.4)$ & $55(67.1)$ & $12(75.0)$ & 0.533 \\
\hline Height, cm & $164.7 \pm 8.7$ & $164.2 \pm 8.4$ & $166.8 \pm 10.2$ & 0.289 \\
\hline Weight, kg & $66.9 \pm 13.4$ & $66.9 \pm 13.1$ & $66.4 \pm 15.3$ & 0.892 \\
\hline BMI, kg/m² & $24.5 \pm 3.6$ & $24.7 \pm 3.5$ & $23.6 \pm 3.8$ & 0.288 \\
\hline $\mathrm{SBP}, \mathrm{mmHg}$ & $127.5 \pm 20.1$ & $125.8 \pm 19.7$ & $136.6 \pm 20.6$ & 0.050 \\
\hline $\mathrm{DBP}, \mathrm{mmHg}$ & $77.2 \pm 14.0$ & $76.8 \pm 14.1$ & $79.4 \pm 13.8$ & 0.488 \\
\hline Hypertension, n (\%) & $82(83.7)$ & $67(81.7)$ & $15(93.8)$ & 0.233 \\
\hline Diabetes mellitus, n (\%) & $38(38.8)$ & $33(40.2)$ & 5 (31.3) & 0.499 \\
\hline CKD, n (\%) & $23(23.5)$ & $17(20.7)$ & $6(37.5)$ & 0.148 \\
\hline $\mathrm{CHF}, \mathrm{n}(\%)$ & $28(28.6)$ & $18(22.0)$ & $10(62.5)$ & 0.001 \\
\hline Stroke, n (\%) & $46(46.9)$ & $39(47.6)$ & $7(43.8)$ & 0.780 \\
\hline History of CAD or PAD, $n(\%)$ & $46(46.9)$ & $37(45.1)$ & $9(56.3)$ & 0.415 \\
\hline $\mathrm{CHA}_{2} \mathrm{DS}_{2}$-VASc score & $4.3 \pm 1.6$ & $4.3 \pm 1.6$ & $4.5 \pm 1.7$ & 0.596 \\
\hline LVEDD, mm & $50.1 \pm 5.6$ & $49.3 \pm 4.9$ & $54.3 \pm 7.0$ & 0.001 \\
\hline LVESD, mm & $34.3 \pm 5.8$ & $33.6 \pm 5.4$ & $38.0 \pm 6.8$ & 0.005 \\
\hline LV mass index, $\mathrm{g} / \mathrm{m}^{2}$ & $109.9 \pm 30.2$ & $103.3 \pm 21.1$ & $144.0 \pm 44.8$ & 0.003 \\
\hline LVEF, \% & $60.7 \pm 11.6$ & $61.8 \pm 10.3$ & $55.4 \pm 16.2$ & 0.146 \\
\hline $\mathrm{LA}$ volume index, $\mathrm{mL} / \mathrm{m}^{2}$ & $57.3 \pm 23.8$ & $54.3 \pm 21.4$ & $72.9 \pm 29.7$ & 0.004 \\
\hline E velocity, $\mathrm{m} / \mathrm{s}$ & $0.87 \pm 0.2$ & $0.85 \pm 0.2$ & $0.95 \pm 0.3$ & 0.127 \\
\hline Deceleration time, ms & $181.4 \pm 77.3$ & $179.5 \pm 78.9$ & $191.0 \pm 70.4$ & 0.589 \\
\hline $\mathrm{S}^{\prime}$ velocity, $\mathrm{cm} / \mathrm{s}$ & $5.4 \pm 1.6$ & $5.4 \pm 1.6$ & $5.4 \pm 1.9$ & 0.962 \\
\hline $\mathrm{e}^{\prime}$ velocity, $\mathrm{cm} / \mathrm{s}$ & $6.4 \pm 1.8$ & $6.5 \pm 1.8$ & $5.8 \pm 1.6$ & 0.130 \\
\hline$E / e^{\prime}$ & $14.7 \pm 6.1$ & $14.0 \pm 5.7$ & $17.9 \pm 7.0$ & 0.020 \\
\hline $\mathrm{RVSP}, \mathrm{mmHg}$ & $32.6 \pm 10.5$ & $31.3 \pm 9.2$ & $39.8 \pm 13.8$ & 0.029 \\
\hline LA reservoir stain, $\%$ & $14.3 \pm 6.5$ & $15.3 \pm 6.6$ & $9.2 \pm 3.4$ & $<0.001$ \\
\hline
\end{tabular}

Data are presented as mean $( \pm \mathrm{SD})$ or $\mathrm{n}(\%)$. BMI, body mass index; CAD, coronary artery disease; CHF, congestive heart failure; CKD, chronic kidney disease; DBP, diastolic blood pressure; E, mitral inflow early diastolic filling; e', early diastolic mitral annular tissue velocity; HF, heart failure; LA, left atrium; LAAO, left atrial appendage occlusion; LV, left ventricle; LVEF, left ventricular ejection fraction; LVEDD, left ventricular end-diastolic dimension; LVESD, left ventricular end-systolic dimension; PAD, peripheral artery disease; RVSP, right ventricular systolic pressure; S', systolic mitral annular tissue velocity; SBP, systolic blood pressure.

ventricular (LV) ejection fraction, LA volume index (LAVI), and LV mass index (LVMI) were used for covariates. A maximum caliper width of 0.2 was used. To ensure balance and reduce the bias, absolute standardized mean differences $<0.2$ were considered as indicators. The study was conducted in accordance with the Declaration of Helsinki, and the study protocol was approved by the Institutional Review Board of the Yonsei University Health System.

\section{Follow up and Clinical Outcomes}

After LAAO, all patients were followed routinely at the outpatient clinic at 1 month, 6 months, and 1-year intervals after the procedure. The primary endpoint was unplanned hospital admission due to HF. Hospital HF admission was defined when the following conditions were met: dyspnea symptoms typically at a minimum of New York Heart Association (NYHA) class 3 with required medical treatment such as intravenous diuretics, elevated $\mathrm{N}$-terminal pro-B-type natriuretic peptide (NT-proBNP), and pulmonary edema or pleural effusion on chest X-ray.

\section{Conventional Echocardiography}

Standard 2-dimensional (2D) and Doppler measurements were performed in all patients under the guidelines of the
American Society of Echocardiography. ${ }^{\mathbf{1 1}}$ Both LAVI and LVMI were measured using the modified Simpson's method with apical 4- and 2-chamber views and indexed on the basis of body surface area. Diastolic parameters such as peak velocity of early diastolic mitral inflow, deceleration time of E velocity, and early velocity in diastolic mitral annulus of septum (e') were measured using pulse-wave and tissue Doppler. E/e' was calculated and used as an index of LV filling pressures. Right ventricular systolic pressure (RVSP) was estimated by summing the peak systolic pressure from the maximal tricuspid regurgitation jet velocity using the modified Bernoulli's equation, and right atrial pressure was estimated using the diameter and collapsibility of the inferior vena cava (IVC).

\section{Speckle-Tracking Echocardiography}

2D speckle-tracking echocardiography was performed for the longitudinal deformation variables of the LA according to the guidelines of the joint European Association of Cardiovascular Imaging and American Society of Echocardiography. ${ }^{12}$ The apical 4-chamber view for each echocardiographic image was stored offline for at least 3 consecutive cycles at a frame rate of 50-70 frames/s. Strain analysis was performed using vendor-independent 2D 


\begin{tabular}{|c|c|c|c|c|}
\hline & \multicolumn{2}{|c|}{ Univariate analysis } & \multicolumn{2}{|c|}{ Multivariate analysis } \\
\hline & $\begin{array}{c}\text { Hazard ratio } \\
{[95 \% \mathrm{Cl}]}\end{array}$ & $P$ value & $\begin{array}{c}\text { Hazard ratio } \\
{[95 \% \mathrm{Cl}]}\end{array}$ & $P$ value \\
\hline Age & 0.99 [0.94-1.04] & 0.606 & & \\
\hline Male sex & $1.54[0.50-4.79]$ & 0.456 & & \\
\hline History of CHF & $6.07[2.17-16.9]$ & 0.001 & $4.78[1.55-14.7]$ & 0.006 \\
\hline LA reservoir strain & $0.78[0.68-0.90]$ & 0.001 & $0.80[0.70-0.93]$ & 0.003 \\
\hline LA volume index & $1.03[1.01-1.04]$ & 0.002 & & \\
\hline$E / e^{\prime}$ & $1.09[1.03-1.16]$ & 0.003 & $1.11[1.02-1.20]$ & 0.014 \\
\hline RVSP & $1.08[1.04-1.12]$ & $<0.001$ & & \\
\hline LV mass index & $1.03[1.02-1.04]$ & $<0.001$ & $1.02[1.00-1.03]$ & 0.023 \\
\hline LVEF & 0.96 [0.93-1.00] & 0.029 & & \\
\hline
\end{tabular}

Abbreviations as in Table 1.

strain software (Cardiac Performance Analysis software; TomTec). To define the region of interest (ROI), tracing of the LA endocardium began at the septal area of the mitral annulus and continued through the LA endocardial border, without including the adjacent pericardial tissue. It traveled across the pulmonary veins and LAA orifice and finally ended at the lateral area of the mitral annulus. Then, a composite LA longitudinal strain in the whole cardiac cycle was calculated. The focus was positioned at an intermediate depth, and the width of the ROI was manually adjusted to encompass the thickness of the LA myocardium. If the foreshortened images were obtained on TTE or tracking quality was poor, those images were excluded from the strain assessment.

To calculate the LA strains, the onset of the QRS complex was set to 0 references. Each LA strain was defined as follows: LA reservoir strain was the peak longitudinal LA strain between the onset of the QRS complex and the peak of the $\mathrm{T}$ wave. Because all of the patients had AF, there was no coordinated LA contraction; therefore, the LA conduit strain, which was the result of subtracting the LA contraction strain from the LA reservoir strain in sinus rhythm, remained the same as the LA reservoir strain. The data of the LA strain were carefully reviewed by 2 expert cardiologists who were blinded to the clinical information.

\section{Statistical Analysis}

Continuous variables are presented as mean \pm standard deviation, and categorical variables are presented as frequency and percentage. Baseline patients' clinical and echocardiographic characteristics were analyzed using the Student's t-test for continuous variables and Chi-squared $\left(\chi^{2}\right)$ test for categorical variables. Prognostic factors of hospital HF admission were evaluated using a multivariate Cox proportional regression analysis on forward stepwise selection from the clinical variables. All variables with $\mathrm{P}$ values $<0.10$ in univariate analysis were included first, and then the variables were analyzed using multivariable Cox analysis. Cut-off points of the independent prognostic indicators for hospital $\mathrm{HF}$ admission were calculated using receiver operating characteristic (ROC) curve analysis, and the optimal cut-off points were selected by maximizing sensitivity and specificity across various cut-off points. The comparison among ROC curves was made by using De Long's test. Time-dependent ROC curve analyses were also performed and reported the associated area under the curves (AUC) at years 1 to 4 . The rate of hospital $\mathrm{HF}$ admission was estimated using Kaplan-Meier survival analysis, and the groups were compared using a log-rank test. In subgroup analysis, Kaplan-Meier survival analyses were compared using a stratified log-rank test. $\mathrm{P}$ values $<0.05$ were considered statistically significant.

\section{Results}

\section{Baseline Clinical Characteristics and Echocardiographic Variables}

Of the 106 enrolled patients who had LAAO, 8 patients were excluded because of absence or poor imaging quality of the baseline echocardiography. A total of 98 patients (mean age $70 \pm 9$ years, $68 \%$ male) were included and followed for a mean follow-up duration of $36 \pm 26$ months. During follow up, $16(16 \%)$ patients experienced hospital $\mathrm{HF}$ admission after LAAO. Baseline clinical characteristics and echocardiographic variables of the study population are summarized in Table 1. In patients with HF admission, a previous history of HF was more common than in those who did not experience hospital HF admission (63\% vs. $22 \%, \mathrm{P}=0.001$ ). Among the echocardiographic parameters, patients who had admitted for HF had larger LA (LAVI $73 \pm 30$ vs. $54 \pm 21 \mathrm{~mL} / \mathrm{m}^{2}, \mathrm{P}=0.004$ ) and LV (LVMI $144 \pm 45$ vs. $\left.103 \pm 21 \mathrm{~g} / \mathrm{m}^{2}, \mathrm{P}=0.003\right)$ and had more severe diastolic dysfunction (RVSP $40 \pm 14$ vs. $31 \pm 9 \mathrm{mmHg}, \mathrm{P}=0.029$; E/e' $18 \pm 7$ vs. $14 \pm 6, P=0.020$ ). In speckle-tracking echocardiographic data, the LA reservoir strain was significantly lower in patients who had were admitted for HF ( $9 \pm 3$ vs. $15 \pm 7 \%, \mathrm{P}<0.001)$.

\section{Predictors of Hospital HF Admission After LAAO}

Table 2 shows the factors associated with the development of HF after LAAO. In univariate analysis, a history of HF, lower LA reservoir strain, factors implying severe diastolic dysfunction (e.g., higher LAVI, E/e', and RVSP), higher LVMI, and lower LV ejection fraction were associated with an increased risk of hospital HF admission after LAAO. A multivariate model revealed 4 factors affecting hospital HF admission. A history of $\mathrm{HF}$ (hazard ratio [HR] 4.78, 95\% confidence interval [CI] 1.55-14.7, $\mathrm{P}=0.006$ ), lower LA strain (HR $0.80,95 \%$ CI $0.70-0.93$, $\mathrm{P}=0.003$ ), higher $\mathrm{E} / \mathrm{e}$ ' (HR 1.11, 95\% CI 1.02-1.20, $\mathrm{P}=0.014$ ), and higher LVMI (HR 1.02, 95\% CI 1.00-1.03, $\mathrm{P}=0.023$ ) were identified as significant prognostic factors of hospital HF admission after LAAO. ROC analyses showed the cut-off values for LA reservoir strain as $10 \%$, 


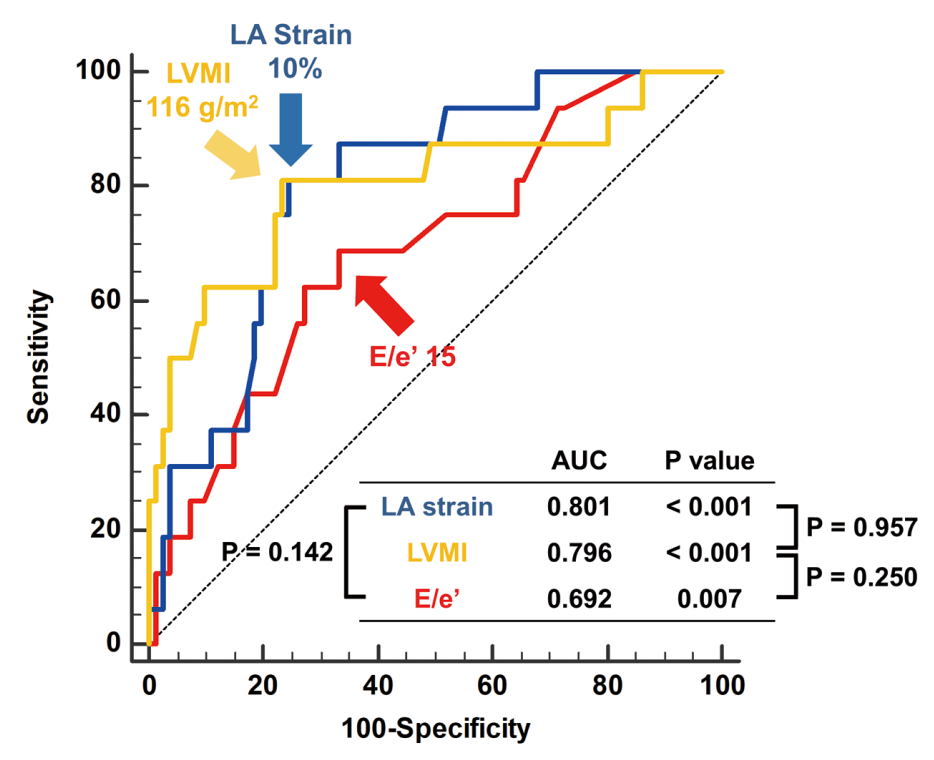

Figure 1. ROC analysis with area under the curve and $P$ values shown for LA strain (blue), LVMI (yellow), and E/e' (red). The cut-off points were determined at each value that showed the maximum likelihood ratio. LA, left atrium; LVMI, left ventricular mass index; ROC, receiver operating characteristic.

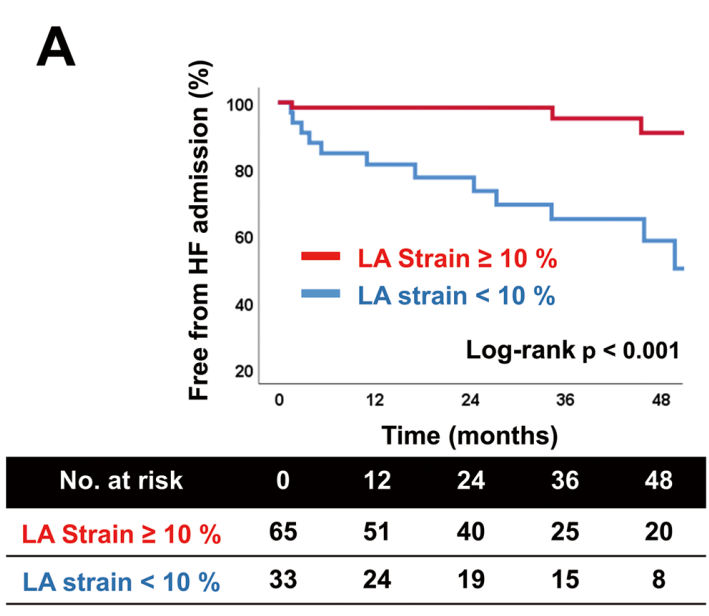

B

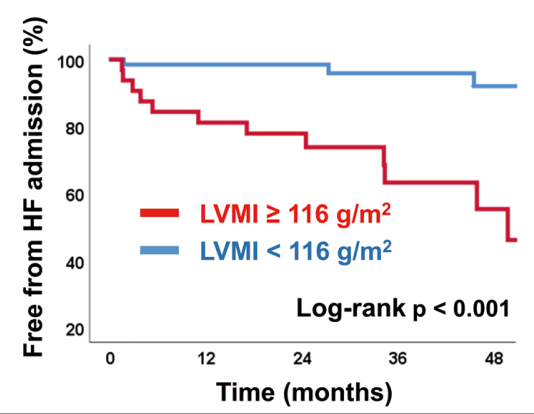

\begin{tabular}{cccccc} 
No. at risk & 0 & 12 & 24 & 36 & 48 \\
\hline LVMI $\geq 116 \mathrm{~g} / \mathrm{m}^{2}$ & 32 & 25 & 19 & 10 & 6 \\
\hline $\mathrm{LVMI}<116 \mathrm{~g} / \mathrm{m}^{2}$ & 66 & 50 & 40 & 30 & 22
\end{tabular}

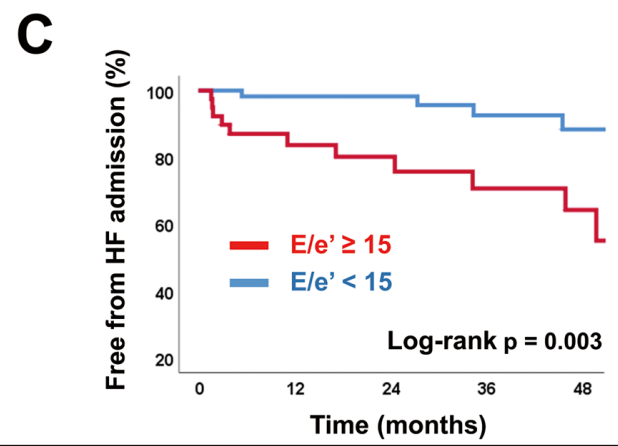

$\begin{array}{llllll}\text { No. at risk } & 0 & 12 & 24 & 36 & 48\end{array}$

\begin{tabular}{cccccc}
$E / e^{\prime} \geq 15$ & 39 & 22 & 18 & 12 & 8 \\
\hline E/e' $<15$ & 59 & 50 & 41 & 28 & 20
\end{tabular}

Figure 2. Kaplan-Meier analyses regarding hospital HF admission as dependent on (A) LA strain, (B) LVMI, and (C) E/e'. HF, heart failure; LA, left atrium; LVMI, left ventricular mass index. 

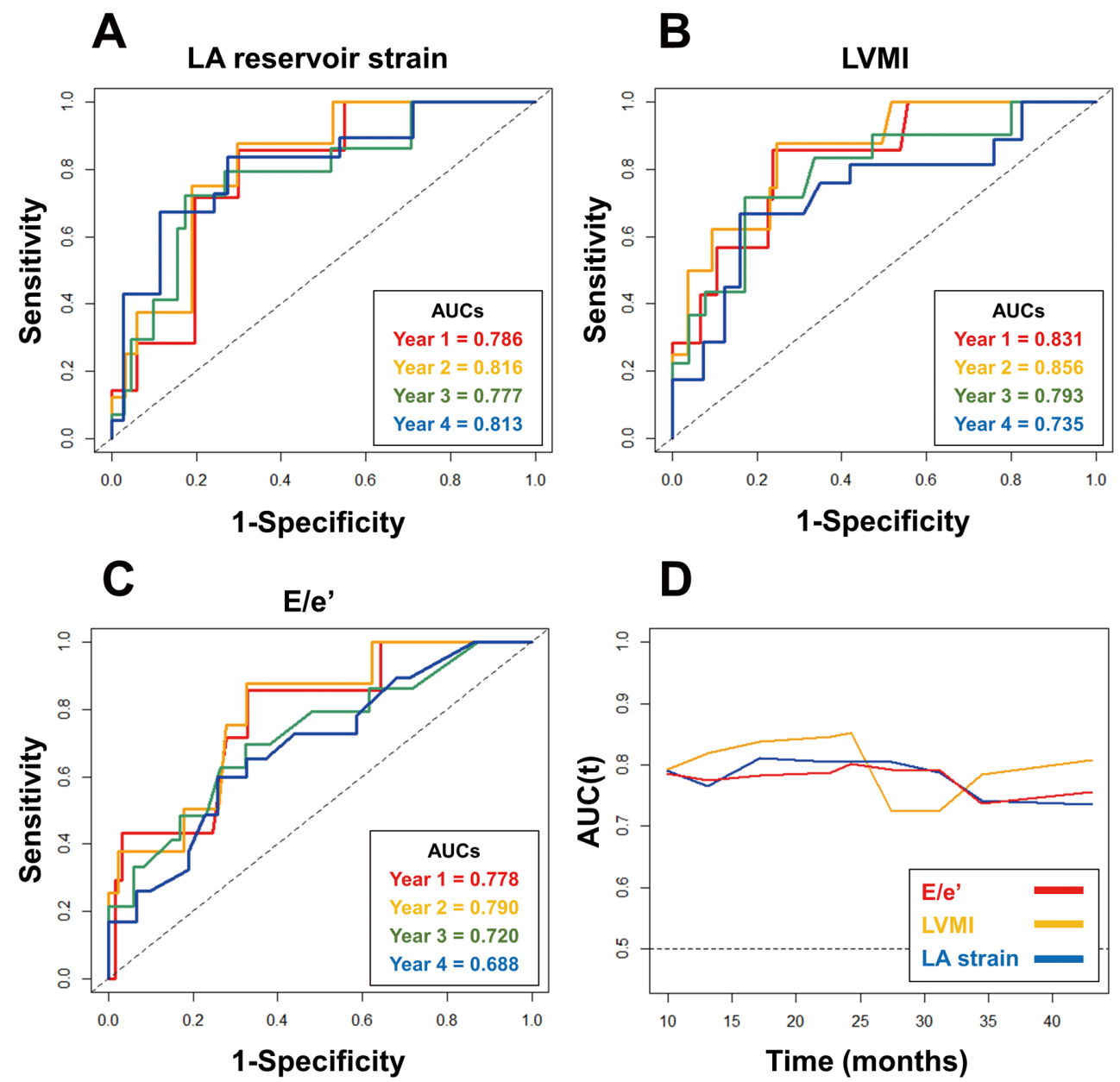

Figure 3. Time-dependent ROC curves representing the accuracy of the (A) LA strain, (B) LVMI, and (C) E/e', and (D) timedependent AUC curves of prediction models for each echocardiographic parameters. The X-axis represented the follow-up months, and the $Y$-axis represented the estimated AUC for survival at specific times of interest. AUC, area under the curve; LA, left atrium; LVMI, left ventricular mass index; ROC, receiver operating characteristic.

LVMI as $116 \mathrm{~g} / \mathrm{m}^{2}$, and E/e' as 15 (Figure 1). Figure 2 shows Kaplan-Meier analyses of hospital HF admission depending on those cut-off values. Patients with lower LA reservoir strain $(<10 \%)$, higher LVMI $\left(\geq 116 \mathrm{~g} / \mathrm{m}^{2}\right)$, and higher E/e' $(\geq 15)$ were more likely to have a worse outcome than those patients who did not have values beyond the cut-offs for these factors. Time-dependent ROC analyses were additionally produced for years 1 to 4 , due to the censored nature of the echocardiographic variables (Figure 3). In those analyses, the AUC of LA reservoir strain, LVMI, and E/e' in the second year of follow up showed higher results $(0.816,0.856$, and 0.790 , respectively) compared to other years. The addition of LA reservoir strain over both E/e' and LVMI significantly improved the prognostic value in association with hospital HF admission, and the global chi-squared value was increased from 9.7 to 36.2 (Figure 4).

\section{Subgroup Analysis: Propensity-Matched Comparison}

A propensity-matched sample with LAAO $(n=93)$ and without LAAO $(n=93)$ was generated. After matching, there were no significant differences in baseline clinical and echocardiographic characteristics between those with and without LAAO (Table 3). Figure 5 shows the Kaplan-Meier analyses for hospital HF admission and a composite of hospital HF admission and all-cause mortality. Patients with LAAO revealed a significantly higher incidence of subsequent HF than the control group $(\mathrm{P}=0.046)$; however, there was no significant difference in composite of hospital HF admission and all-cause mortality between the groups $(\mathrm{P}=0.083)$. On the Cox regression analysis for determining the factors as a prognostic indicator of subsequent hospital HF admission in a propensity-matched cohort, a multivariate model revealed that LAAO (HR 3.76, CI 1.19$11.88, \mathrm{P}=0.024$ ), along with a history of $\mathrm{HF}$ (HR 3.78, CI 1.27-11.24, P=0.017) and higher LVMI (HR 1.02, CI $1.00-1.03, \mathrm{P}=0.015$ ), were associated with an increased risk of hospital HF admission (Supplementary Table).

\section{Discussion}

The principal findings of the present study are as follows: (1) subsequent hospital HF admission was more frequent 


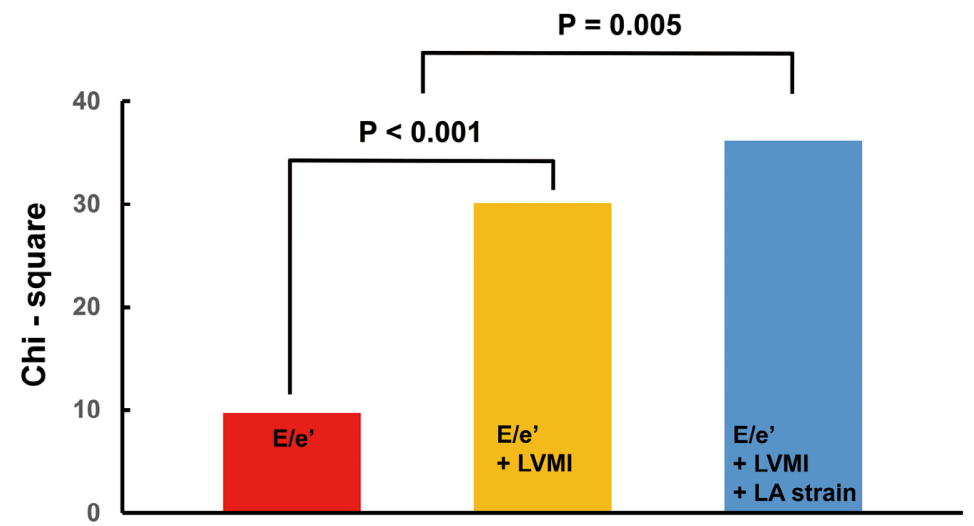

Figure 4. Incremental prognostic value of LA strain over E/e' and LVMI. LA, left atrium; LVMI, left ventricular mass index.

Table 3. Baseline Characteristics and Echocardiographic Variables of the Propensity Matched Sample

\begin{tabular}{lccc} 
& $\begin{array}{c}\text { LAAO } \\
(\mathbf{N = 9 3 )}\end{array}$ & $\begin{array}{c}\text { Control } \\
\mathbf{( N = 9 3 )}\end{array}$ & P value \\
Age, years & $70.6 \pm 9.0$ & $69.9 \pm 9.8$ & 0.630 \\
Male sex, $\mathrm{n}(\%)$ & $62(66.7)$ & $65(69.9)$ & 0.636 \\
Hypertension, $\mathrm{n}(\%)$ & $77(82.8)$ & $71(76.3)$ & 0.275 \\
Diabetes mellitus, $\mathrm{n}(\%)$ & $35(37.6)$ & $28(30.1)$ & 0.278 \\
CKD, $\mathrm{n}(\%)$ & $20(21.5)$ & $25(26.9)$ & 0.392 \\
CHF, $\mathrm{n}(\%)$ & $26(28.0)$ & $31(33.3)$ & 0.426 \\
Stroke, $\mathrm{n}(\%)$ & $41(44.1)$ & $40(43.0)$ & 0.882 \\
History of CAD or PAD, $\mathrm{n}(\%)$ & $41(44.1)$ & $40(43.0)$ & 0.882 \\
CHA2DS2-VASc score & $4.3 \pm 1.6$ & $4.1 \pm 1.7$ & 0.475 \\
LV mass index, g/m² & $110.3 \pm 30.6$ & $109.6 \pm 30.0$ & 0.879 \\
LVEF, \% & $60.7 \pm 11.7$ & $62.2 \pm 11.6$ & 0.382 \\
LA volume index, $\mathrm{mL} / \mathrm{m}^{2}$ & $57.2 \pm 23.8$ & $56.2 \pm 25.3$ & 0.772 \\
E/e' & $14.4 \pm 5.8$ & $147 \pm 7.5$ & 0.764 \\
LA strain, \% & $14.4 \pm 6.7$ & $14.2 \pm 7.8$ & 0.870 \\
\hline
\end{tabular}

Data are presented as mean $( \pm \mathrm{SD})$ or $\mathrm{n}(\%)$. Abbreviations as in Table 1 .

A

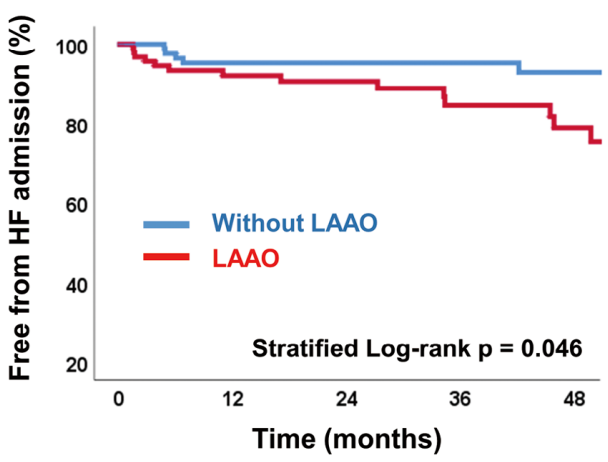

B

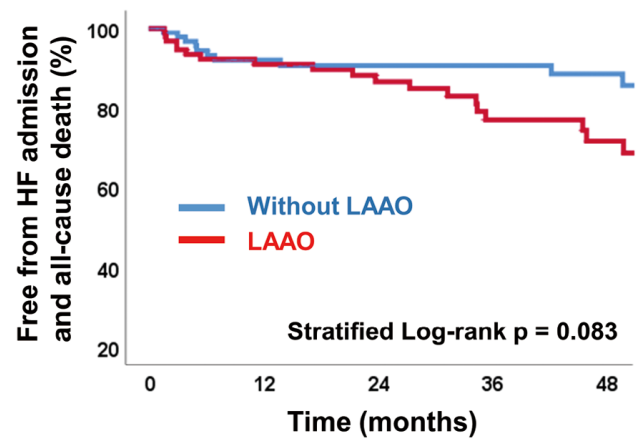

\begin{tabular}{cccccc} 
No. at risk & 0 & 12 & 24 & 36 & 48 \\
\hline Without LAAO & 93 & 69 & 57 & 47 & 33 \\
\hline LAAO & 93 & 70 & 54 & 36 & 25 \\
\hline
\end{tabular}

\begin{tabular}{cccccc} 
No. at risk & 0 & 12 & 24 & 36 & 48 \\
\hline Without LAAO & 93 & 69 & 57 & 47 & 33 \\
\hline LAAO & 93 & 70 & 54 & 36 & 25 \\
\hline
\end{tabular}

Figure 5. Kaplan-Meier analyses of (A) subsequent hospital HF admission and (B) composite of subsequent hospital HF admission and all-cause death in propensity-matched sample between the group of patients who underwent LAAO and those who did not undergo LAAO. HF, heart failure; LAAO, left atrial appendage occlusion. 
in patients who had LAAO when compared with those who did not have LAAO; (2) the incidence of hospital HF admission after LAAO was not uncommon $(16 \%)$ during the mean follow up of 36 months; (3) lower LA reservoir strain, along with a history of HF, higher E/e', and LVMI, were identified as independent predictors of hospital HF admission after LAAO. These findings underscore the importance of preprocedural assessment of these parameters before attempting LAAO.

\section{Incidence of Hospital HF Admission After LAAO}

Development of HF after LAAO has previously been described in a case report, ${ }^{10}$ but it was not consistently assessed in previous trials about LAAO. In this retrospective cohort study, the incidence of subsequent hospital HF admission after LAAO was more frequent compared with propensity-matched controls, and it was reported as $16 \%$ of the total study population of LAAO patients. One study reported that the incidence of HF rehospitalization after LAAO was $17 \%$ ( 6 of the 35 patients) during the $10 \pm 6$ months of follow up. ${ }^{13}$ Because LAA has reservoir function, ${ }^{9}$ exclusion of LAA may result in unfavorable hemodynamics. It has been shown that exclusion of LAA during coronary artery bypass graft surgery was associated with a greater risk of postoperative respiratory failure $(8.2 \% \mathrm{vs}$. $6.2 \%, \mathrm{P}<0.0001)$, acute kidney injury $(21.8 \%$ vs. $18.5 \%$, $\mathrm{P}<0.0001)$, and readmission $(16.0 \%$ vs. $9.5 \%, \mathrm{P}<0.001) .{ }^{14}$ Although a definite cause-and-effect relationship cannot be established, it is plausible that LAA exclusion mitigates the beneficial hormonal and hemodynamic role of the LAA. These results are consistent with our study.

\section{Predictors of HF Development After LAAO}

Predictors of hospital HF admission after LAAO in patients with AF have not been determined in previous studies. In our study, several independent predictors were identified, and these factors reflect the vulnerable substrate for the development of HF. In other words, patients with unfavorable structural remodeling or significant diastolic function with unfavorable hemodynamics are prone to develop HF after LAAO because of a lack of reservoir function of LAA after the procedure. In the presence of cardiac remodeling, patients are susceptible to various volume overloads, which may contribute to being at higher risk for hospital HF admission after LAAO. In a previous study that aimed to demonstrate the impact of LAAO on cardiac functional and structural remodeling, patients showed a significant increase in LV filling pressure (E/e') for 12 months following LAAO when compared with patients without LAAO. ${ }^{15}$ These findings may be explained by poor regulation of volume and pressure caused by the loss of LAA function. Therefore, patients who are already in a state of high LV filling pressure may be vulnerable to developing HF, and it could be supported by the results of our study. The most important prognostic factor of hospital $\mathrm{HF}$ admission was lower LA strain. LAA is 2.6 -fold more compliant than the LA chamber itself, and has the important ability to control instances of elevated LA pressure and decreased LA distensibility. ${ }^{10,16}$ In previous animal studies, a decrease of LA compliance after LAA exclusion was described as an augmented pressure-volume curve and reduction of pulmonary venous flow. ${ }^{17,18}$ It suggests that removal of the LAA will have an unfavorable effect by decreasing LA compliance and reservoir function.

Speckle-tracking LA strain measures are already regarded to be useful for the diagnosis of $\mathrm{HF}$ with preserved ejection fraction. ${ }^{19}$ As LA reservoir function is modulated by LV systolic function, atrial size, and LA compliance, ${ }^{20}$ decreased values of LA strain are related to lower LA compliance. In a speckle-tracking imaging study, LA strain was significantly reduced in early diastolic dysfunction, which was associated with higher LA stiffness and LA size, which are this coincides with decreased LA compliance. ${ }^{21}$ Decreased function of the LA, which is expressed as decreased LA strain, is an important prognostic factor for the occurrence of $\mathrm{HF},{ }^{22}$ and its importance could be applied to our study for predicting the risk of HF in a state of decreased LA and diastolic function.

Structural and functional evaluation of LA and LV should be further emphasized to identify individuals who would receive maximal benefits from LAAO. This is because AF is not just a risk for systemic embolism, and LAAO may not improve LA and LV hemodynamics. In particular, if patients have structural remodeling and elevated LV filling pressure and abnormal LA strain, their risks for developing HF will increase despite a successful LAAO. The presence of HF history, LV hypertrophy, significant diastolic dysfunction, and decreased LA strain may help identify a cohort of patients with AF for whom LAAO has limited long-term benefits.

\section{Study Limitations}

This study had some limitations. First, this study was retrospectively performed in a single center, and there were no available data on regular follow up related to cardiac symptoms after LAAO. Second, the number of enrolled patients was relatively low $(\mathrm{n}=98)$, so large-scaled prospective studies of patients with LAAO are needed. Third, the duration of clinical follow up in this study was relatively short (36 months), which may be not sufficient to evaluate the impact of LAAO on the occurrence of subsequent HF. Finally, although a higher frequency of hospital HF admission was demonstrated in patients with LAAO through comparison with the propensity-matched sample, the possibility of non-corrected selection bias remains, which could not be corrected.

\section{Conclusions}

LAAO increases HF events, and it is not uncommon even after successful LAAO; therefore, the development of HF should be assessed in patients who have undergone LAAO. Because a history of HF, LA function, and diastolic function are independently associated with the development of $\mathrm{HF}$, these factors should be considered before attempting LAAO.

\section{Disclosures}

The authors have no conflicts of interests to declare.

\section{Author Contributions}

D.-Y.K.: Data analysis, collection, and drafting the manuscript. M.J.K., J.S., I.C.: Data collection and revising it critically for important intellectual content.

C.Y.S., G.-R.H.: Data analysis, collection, and revising it critically for important intellectual content.

J.-S.K.: Data analysis and revising it critically for important intellectual content.

J.-W.H.: Corresponding author. Conception and design of the study, drafting the manuscript, and revising it critically for important intellectual content. 


\section{Data Availability}

It was not possible to share the de-identified participant data because the local IRB deliberation did not approve data sharing.

\section{IRB Information}

This study was approved by Yonsei University Health System (IRB number: 4-2020-1268).

\section{References}

1. Lippi G, Sanchis-Gomar F, Cervellin G. Global epidemiology of atrial fibrillation: An increasing epidemic and public health challenge. Int J Stroke 2021; 16: 217-221.

2. Yagi N, Suzuki S, Nagai K, Tanaka T, Nagahama T, Arita T, et al. Current status of oral anticoagulant adherence in Japanese patients with atrial fibrillation: A claims database analysis. $J$ Cardiol 2021; 78: 150-156.

3. Hart RG, Benavente O, McBride R, Pearce LA. Antithrombotic therapy to prevent stroke in patients with atrial fibrillation: A meta-analysis. Ann Intern Med 1999; 131: 492-501.

4. Packer M. A compelling case for less aggressive arrhythmia management in patients with chronic heart failure and longstanding atrial fibrillation. J Card Fail 2020; 26: 85-92.

5. Blackshear JL, Odell JA. Appendage obliteration to reduce stroke in cardiac surgical patients with atrial fibrillation. Ann Thorac Surg 1996; 61: 755-759.

6. Harada M. 2-year outcomes of left atrial appendage occlusion with WATCHMAN in Japanese atrial fibrillation patients. Circ J 2020; 84: 1227-1229.

7. Ponikowski P, Voors AA, Anker SD, Bueno H, Cleland JGF, Coats AJS, et al. 2016 ESC Guidelines for the diagnosis and treatment of acute and chronic heart failure: The Task Force for the diagnosis and treatment of acute and chronic heart failure of the European Society of Cardiology (ESC) developed with the special contribution of the Heart Failure Association (HFA) of the ESC. Eur Heart J 2016; 37: 2129-2200.

8. Kemi Y, Yamashita E, Fujiwara T, Kario K, Sasaki T, Minami $\mathrm{K}$, et al. The prevalence and characteristics of candidates for percutaneous left atrial appendage occlusion using a WATCHMAN device among patients who underwent atrial fibrillation ablation in a Japanese population. $J$ Echocardiogr 2021; 19: 243-249.

9. Stöllberger C, Schneider B, Finsterer J. Elimination of the left atrial appendage to prevent stroke or embolism?: Anatomic, physiologic, and pathophysiologic considerations. Chest 2003; 124: $2356-2362$.

10. Schneider B, Nazarenus D, Stöllberger C. A 79-year-old woman with atrial fibrillation and new onset of heart failure. ESC Hear Fail 2019; 6: 570-574.

11. Lang RM, Badano LP, Mor-Avi V, Afilalo J, Armstrong A, Ernande L, et al. Recommendations for cardiac chamber quantifi- cation by echocardiography in adults: An update from the American Society of Echocardiography and the European Association of Cardiovascular Imaging. Eur Heart J Cardiovasc Imaging 2015; 16: $233-271$.

12. Badano LP, Kolias TJ, Muraru D, Abraham TP, Aurigemma G, Edvardsen T, et al. Standardization of left atrial, right ventricular, and right atrial deformation imaging using two-dimensional speckle tracking echocardiography: A consensus document of the EACVI/ ASE/Industry Task Force to standardize deformation imaging. Eur Heart J Cardiovasc Imaging 2018; 19: 591-600.

13. Granier M, Laugaudin G, Massin F, Cade S, Winum PF, Freitag $\mathrm{C}$, et al. Occurrence of incomplete endothelialization causing residual permeability after left atrial appendage closure. $J$ Invasive Cardiol 2018; 30: 245-250.

14. Mahmood E, Matyal R, Mahmood F, Xu X, Sharkey A, Chaudhary $\mathrm{O}$, et al. Impact of left atrial appendage exclusion on short-term outcomes in isolated coronary artery bypass graft surgery. Circulation 2020; 142: 20-28.

15. Phan QT, Shin SY, Cho IS, Lee WS, Won H, Sharmin S, et al. Impact of left atrial appendage closure on cardiac functional and structural remodeling: A difference-in-difference analysis of propensity score matched samples. Cardiol J 2019; 26: 519-528.

16. Davis CA 3rd, Rembert JC, Greenfield JC Jr. Compliance of left atrium with and without left atrium appendage. Am J Physiol Circ Physiol 1990; 259: H1006-H1008.

17. Hoit BD, Shao Y, Tsai LM, Patel R, Gabel M, Walsh RA. Altered left atrial compliance after atrial appendectomy: Influence on left atrial and ventricular filling. Circ Res 1993; 72: 167-175.

18. Kamohara K, Popović ZB, Daimon M, Martin M, Ootaki Y, Akiyama M, et al. Impact of left atrial appendage exclusion on left atrial function. J Thorac Cardiovasc Surg 2007; 133: 174-181.

19. Freed BH, Daruwalla V, Cheng JY, Aguilar FG, Beussink L, Choi A, et al. Prognostic utility and clinical significance of cardiac mechanics in heart failure with preserved ejection fraction: Importance of left atrial strain. Circ Cardiovasc Imaging 2016; 9: $1-10$.

20. Pathan F, D'Elia N, Nolan MT, Marwick TH, Negishi K. Normal ranges of left atrial strain by speckle-tracking echocardiography: A systematic review and meta-analysis. J Am Soc Echocardiogr 2017; 30: 59-70.e8.

21. Khan UA, Simone G De, Hill J, Tighe DA, Aurigemma GP. Depressed atrial function in diastolic dysfunction: A speckle tracking imaging study. Echocardiography 2013; 30: 309-316.

22. Yuda S. Current clinical applications of speckle tracking echocardiography for assessment of left atrial function. $J$ Echocardiogr 2021; 19: 129-140.

\section{Supplementary Files}

Please find supplementary file(s);

http://dx.doi.org/10.1253/circj.CJ-21-0642 